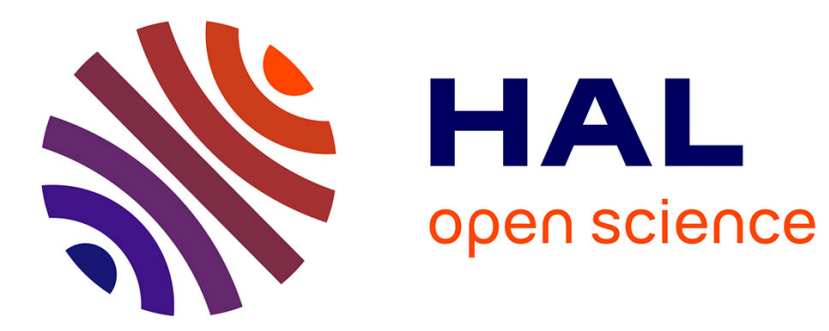

\title{
Terahertz Time-of-Flight Tomography Beyond the Axial Resolution Limit: Autoregressive Spectral Estimation Based on the Modified Covariance Method
}

\author{
Min Zhai, A. Locquet, Cyrielle Roquelet, D. S Citrin
}

\section{- To cite this version:}

Min Zhai, A. Locquet, Cyrielle Roquelet, D. S Citrin. Terahertz Time-of-Flight Tomography Beyond the Axial Resolution Limit: Autoregressive Spectral Estimation Based on the Modified Covariance Method. Journal of Infrared, Millimeter and Terahertz Waves, 2020, 41 (8), pp.926-939. 10.1007/s10762-020-00722-1 . hal-02993335

\section{HAL Id: hal-02993335 \\ https://hal.science/hal-02993335}

Submitted on 18 Nov 2020

HAL is a multi-disciplinary open access archive for the deposit and dissemination of scientific research documents, whether they are published or not. The documents may come from teaching and research institutions in France or abroad, or from public or private research centers.
L'archive ouverte pluridisciplinaire HAL, est destinée au dépôt et à la diffusion de documents scientifiques de niveau recherche, publiés ou non, émanant des établissements d'enseignement et de recherche français ou étrangers, des laboratoires publics ou privés. 


\title{
Terahertz time-of-flight tomography beyond the axial- resolution limit: Autoregressive spectral estimation based on the modified covariance method
}

\author{
Min Zhai ${ }^{1,2}$, Alexandre Locquet ${ }^{1,2}$, Cyrielle Roquelet ${ }^{3}$, D.S. Citrin ${ }^{1,2}$
}

\begin{abstract}
We present a method for exceeding the naïve axial (i.e., depth) resolution limit of terahertz (THz) deconvolution by autoregressive spectral extrapolation (AR) based on the modified covariance method (AR/MCM). In contrast to Wiener filtering combined with wavelet denoising, AR/MCM does not discard any frequency components in the low signal-to-noise (SNR) regions of the measured data, and unlike the AR approach based on the Burg method (AR/BM), neither peak-splitting (single peaks in the impulse response function appearing as double peaks) nor frequency bias (spectral peaks shifted with respect to their correct positions) is observed after deconvolution. After verifying the advantages of AR/MCM over Wiener filtering in conjunction with wavelet denoising as well as over AR/BM, using synthetic data, AR/MCM is employed to reconstruct a single layer of mill scale on a steel coupon from experimental THz time-of-flight tomography data. The reconstruction shows good agreement with the film thickness obtained from destructive cross-sectional measurements. In addition, unlike AR/BM, optimizing the parameters to obtain stable reconstruction is straightforward relying on Akaike's information criterion. This suggeststhat AR/MCM can be easier to implement for $\mathrm{THz}$ nondestructive characterization of stratigraphy under noisy conditions, particularly when estimates of the stratigraphy may not a priori be available.
\end{abstract}

Keywords Terahertz imaging; Autoregressive spectral extrapolation; Burg method; Modified covariance method; Deconvolution; Pulsed terahertz tomography.

\section{Introduction}

The terahertz $(\mathrm{THz})$ electromagnetic band $(100 \mathrm{GHz}-10 \mathrm{THz})$ is situated between millimeter waves and the mid-infrared regions of the electromagnetic spectrum. Terahertz time-of-flight (TOF) tomography is a noninvasive, noncontact, nondestructive, and nonionizing method to characterize quantitatively the structure of numerous nonelectrically conducting materials that may be opaque at visible and infrared wavelengths, or relatively transparent to $\mathrm{x}$-rays. Currently, the approach has attracted considerable attention for various applications, including coating thickness determination [1]-[3], archeology and art [4][6], security [7], material characterization [8][10], and quality control [10][12].

In general, the stratigraphy of a layered sample subjected to an incident roughly single-cycle $\mathrm{THz}$ pulse can be quantitatively measured based on the arrival time of reflected pulses (henceforth called echoes) reflected from internal and external interfaces presenting dielectric discontinuities. When the thickness of an individual layer is optically thin in $\mathrm{THz}$ regime [physical thickness divided by the refractive index less than about half the minimum wavelength in the high signal-to-noise (SNR) band]; however, the reflected echoes will temporally overlap, rendering the individual echoes visually indistinct. Therefore, signal-processing techniques may be required to separate the overlapping echoes and extract their precise arrival times from which the thicknesses of the individual layers can then be reconstructed.

In other words, what we desire from such measurements is the reflective impulse-response function $h(t)$, which is intrinsic to the sample structure and does not depend on the temporal pulse shape used to interrogate the sample. For a layered structure with

\author{
D.S. Citrin \\ david.citrin@ece.gatech.edu \\ Min Zhai \\ mzhai6@gatech.edu \\ Alexandre Locquet \\ alexandre@gatech.edu \\ Cyrielle Roquelet \\ cyrielle.roquelet@arcelormittal.com \\ 1 School of Electrical and Computer Engineering, Georgia Institute of Technology, Atlanta, Georgia 30332-0250 USA \\ 2 Georgia Tech-CNRS UMI2958, Georgia Tech Lorraine, 2 Rue Marconi, 57070 Metz, France \\ ArcelorMittal Maizières Research SA, Voie Romaine, 57280 Maizières-lès-Metz, France.
}


abrupt interfaces, $h(t)$ can be approximated as a temporal sequences of Dirac $\delta$-functions corresponding to the various interfaces (as well as accounting for multiple reflections, but these are typically weak and will not be considered here), the amplitudes of which are determined by the Fresnel coefficients of those interfaces. Naïvely, we can obtain the Fourier transform of $h(t)$, i.e., the transfer function $H(\omega)$ with $\omega$ the angular frequency, as the ratio of the Fourier transforms of the reflected to the incident electricfield amplitudes. In practice, however, noise may severely limit the useable bandwidth of $H(\omega)$, and introduce anomalous peaks in the reconstructed $h(t)$ after inverse Fourier transformation. The conventional denoising approach is to apply a frequency-domain filter to eliminate high-frequency noise. Double-Gaussian [14], van Hann [15], and Gaussian filters [16] are commonly selected. Clearly, such approaches can only reduce the bandwidth in which $H(\omega)$ is reliably computed, negatively impacting the axial resolution and thus the ability to analyze optically thin layers. Mittleman first suggested employing wavelet transforms for $\mathrm{THz}$ signal processing because $\mathrm{THz}$ pulses as produced may be similar in form to common wavelet basis functions [17]. In contrast to the Fourier transform, which employs an infinite set of sinusoids with frequency ranging from 0 to $\infty$ as basis functions, the wavelet transform employing a truncated basis may be a more efficient representation of a THz pulse because of the time-frequency localization of the wavelet basis function [18]. Chen coupled wavelet denoising with a double-Gaussian inverse filter and achieved high resolution for $h(t)$ [16] and Ferguson and Abbott applied wavelet techniques coupled with Wiener deconvolution to successfully remove Gaussian white noise [19][20]. Those filters further narrow the bandwidth of $H(\omega)$ by eliminating the lowSNR regions, which also contain information about the sample; therefore, the features in $h(t)$ recovered from the inverse Fourier transform of the filtered $H(\omega)$ are temporally broader than ideal impulses, which consequently limits the axial resolution.

Two methods, autoregressive spectra extrapolation (AR) and sparsity-based deconvolution (SD), have been used by our group and by others to enable the extraction of depth information below the naïve axial-resolution limit of signals from samples with optically thin layers. SD retrieves $h(t)$ in the time domain assuming only a limited number of non-zero values (sparse constraint). SD has improved the axial resolution of THz TOF tomography based on the iterative shrinkage algorithm [21][23]. In Ref. [24], SD quantitatively revealed the detailed stratigraphy of a $17^{\text {th }}$ century easel painting with some layer thicknesses less than $50 \mu \mathrm{m}$. Computation time, however, can be long, making it arduous to handle large data sets. Autoregressive spectral extrapolation (AR), applied previously in geophysics [25] and then extended to acoustics [26][27], enhances the ability to distinguish thin layers by extrapolating the data into the low-SNR bands based on the data within the high-SNR region using techniques from time-series prediction, though applied in the frequency domain. In other words, by supplementing the experimental data with expectations concerning the information-theoretic complexity of $H(\omega)$, one can achieve super-resolution in the axial direction. In Ref. [28], Dong et al. reconstructed $h(t)$ of a single-layer polymer coating of thickness $22.5 \mu \mathrm{m}$ using AR based on the Burg method (AR/BM). Although AR/BM can exceed the naïve axial resolution limit, it may suffer from temporal peak splitting and frequency bias when the order of AR model is too large, as reported in Refs. [29][30] and discussed below. This limits the applicability of $\mathrm{AR} / \mathrm{BM}$ without prior knowledge of the sample structure [31]. Moreover, AR/BM we observe that can be difficult to implement insofar as finding parameters providing accurate and robust reconstruction.

In this paper, $\mathrm{AR}$ based on the modified covariance method (AR/MCM) is proposed to provide super-resolution reconstruction for optically thin layers applied to pulsed THz TOF tomography while suppressing artifacts that may arise in AR/BM as well as easing the choice of parameters associated with AR. In contrast with AR/BM as well as with Wiener filtering combined with wavelet denoising, AR/MCM shows better performance in providing super-resolution capability combined with suppression of artifacts given noisy data. Indeed, unlike the former two approaches, AR/MCM exhibits a relative absence of pulse broadening and peak splitting. In order to validate the efficiency of AR/MCM to measure the thickness of an optically thin film, a mill-scale layer on steel [32] is investigated by this method. The thickness calculated based on AR/MCM shows excellent agreement with destructive measurements obtained by cross-sectional imaging, while being relatively easy to perform compared with AR/BM.

\section{Principle}

We start with a description of the general AR process. Assume frequency $H_{i}$ within a window $H_{i_{L}}<H_{i}<H_{i_{H}}$, where $H_{i_{L}}$ and $H_{i_{H}}$ define the upper- and lower-frequency limits of that window. The AR model serves as a prediction filter to find data components outside the window for $i>i_{H}$ using the forward-prediction equation

$$
\widehat{H}_{i}=-\sum_{k=1}^{p} a_{k} H_{i-k} \text {. }
$$

Similarity, the backward-prediction filter is used to find the missing component for $i<i_{L}$,

$$
\widehat{H}_{i}=-\sum_{k=1}^{p} b_{k} H_{i+k} \text {, }
$$

where $p$ is the order of the AR process, and $a_{k}$ and $b_{k}$ are the coefficients of the AR forward- and backward-prediction filters, respectively.

Choosing an appropriate value for the order $p$ is essential for the success of AR. On the one hand, a too small value means that the model is not sufficiently complex to represent a signal with many subtle important features, meaning that the estimator would be less flexible to capture the underlying trends of spectrum with high SNR. On the other hand, for an excessively large value of $p$, the overly complex model will exaggerate minor fluctuations; in other words, the estimator captures erroneous trends, which may result from noise still present in the high-SNR region. The optimum forward and backward coefficients are determined by minimizing the squared error $\varepsilon_{p}^{2}$ between the model and the available data. In this case, the sum of both the forward- and backwardprediction errors 
is considered.

$$
\varepsilon_{p}^{2}=\sum_{i=i_{L}}^{i_{L}-p}\left|H_{i}-\sum_{k=1}^{p} b_{k} H_{i+k}\right|^{2}+\sum_{i=i_{H}-p}^{i_{H}}\left|H_{i}-\sum_{k=1}^{p} a_{k} \omega_{i-k}\right|^{2}
$$

Akaike's information criterion (AIC), based on the information-theoretic entropy, provides a standard technique to select the order of the AR model [33],[34]. AIC quantifies a compromise between the accuracy and the complexity of the model, and is defined as

$$
\operatorname{AIC}[p]=N \log \left(\varepsilon_{p}^{2}\right)+2 p
$$

where the first term rewards goodness-of-fit while $2 p$ represents an additive penalty term for increasing order, aimed at discouraging overfitting. According to Akaike's theory, the minimum value of AIC corresponds to the best-fit, i.e., the value of $p$ to be used in the AR model.

The two techniques compared in this article, AR/BM and AR/MCM, rely on a minimization of the forward and backward prediction errors in the least squares sense. In the case of $\mathrm{AR} / \mathrm{BM}$, however, the parameters are constrained to satisfy the LevinsonDurbin recursion. In the recursive procedure in AR/MCM, which has the advantage of being computationally efficient, at each step of recursion, a single coefficient is estimated to minimize the sum of the forward and backward prediction errors.

While we have successfully applied both AR/BM and AR/MCM to characterize the thickness of the layers (see below and Ref. [32]), AR/MCM has the distinct advantage that far less effort is needed to find parameters related to the reconstruction algorithm that give accurate results. For AR/BM, though Levinson-Durbin recursion can reduce computation time, we observe that the optimal order $p$ is inconsistent with the value minimizing AIC, which we interpret as resulting from the loss of accuracy introduced by the Levinson-Durbin constraint. Consequently, the reconstructed $h(t)$ based on nonoptimal coefficients may exhibit spurious peaks that misrepresent the stratigraphy, which is of particular concern if we do not have prior information about the structure, in particular if we do not know beforehand the number of layers. For AR/MCM, because no constraint is added to the minimization of the prediction error, the optimal model order $p$ shows a better correspondence with the value that minimizes the AIC.

\section{Experiment}

The measurement in this work is carried out using a pulsed, broadband THz time-domain system from TeraView Ltd (TPS Spectra 3000), shown schematically in Fig. 1. The GaAs photoconductive antenna is excited and produces roughly single-cycle THz pulses with bandwidth extending from $60 \mathrm{GHz}$ to $3 \mathrm{THz}$. The laser used is an Er-doped fibre laser which emits $780 \mathrm{~nm}$ pulses with sub100 femtosecond pulse duration at a repetition rate of $100 \mathrm{MHz}$ and has a time-averaged output power of $>65 \mathrm{~mW}$. THz TOF tomography is performed in reflection mode at almost normal incidence. Before acquiring data, the time domain $\mathrm{THz}$ reference signal produced by the system was recorded by placing a metal plate (excellent reflector) at the sample position. The spectrum of the THz reference signal $f(t)$ is obtained by Fourier transforming the temporal pulse. The sample is raster-scanned by a set of motorized stages moving in the $x$ - and $y$-directions with step size $0.2 \mathrm{~mm}$. Each recorded reflected THz pulse $r(t)$ contains 4096 data points, and the signal is averaged over 10 shots per pixel.

\section{Results and Discussion}

Reconstruction based on synthetic data is first carried out to compare the performance of the three deconvolution techniques: Wiener filtering, $\mathrm{AR} / \mathrm{BM}$, and $\mathrm{AR} / \mathrm{MCM}$. Neglecting material dispersion, an ideal impulse response function $h_{0}(t)$ (the subscript 0 denote synthetic signals) of a simple double layered structure, which contains 4096 data points with the same sampling period $T_{\mathrm{s}}$, is assumed as

$$
h_{0}[n]=\left\{\begin{aligned}
1, & n=1500 \\
0.5, & n=1530 \\
-0.2, & n=1630 \\
0, & \text { otherwise }
\end{aligned}\right.
$$

where $n$ is a discrete-time variable such that $t=n T_{\mathrm{s}}$ (we use $n$ and $t$ interchangeably as convenient) in which the corresponding time delay associated with the two layers is either $30 T_{\mathrm{s}}$ or $100 T_{\mathrm{s}}$. Therefore, for numerical computation, the impulse-response function is built up from discrete-time $\delta$-functions. The reflected signal $r_{0}(t)$ is simulated from the convolution between the reference signal $f(t)$ and the synthetic impulse response function $h_{0}(t)$ together with additive uncorrelated Gaussian white noise $e_{\mathrm{n}}(t)$, i.e., $r_{0}(t)=f(t) \otimes h_{0}(t)+e_{n}(t)$, where $\otimes$ denotes the convolution operator.

Wiener filtering with wavelet denoising: Figure 2 shows the synthesized impulse response function $h_{0}(t)$ (red) and reflected signal $r_{0}(t)$ obtained by convolution with $f(t)$ and subsequently adding with noise $e_{n}(t)$. As is evident in the figure, the reflected echoes temporally overlap and cannot be visually distinguished in the computed raw reflected signal $r_{0}(t)$, owing to the short time interval between echoes arising from two closely-spaced interfaces. In order to separate the overlapping echoes, a combination of Wiener filtering and wavelet decomposition is first applied to attempt to reconstruct the impulse response function $h_{0}(t)$. The reconstructed transfer function is

$$
\widehat{H}(\omega)=\frac{R(\omega) F^{*}(\omega)}{|F(\omega)|^{2}+Q^{2}},
$$


where the asterisk denotes complex conjugation. Here, $R(\omega)$ and $F(\omega)$ correspond to the Fourier transforms of $r_{0}(t)$ and $f_{0}(t)$, respectively, with $\omega$ the angular frequency. The Wiener filter acts like a noise-desensitizing factor $Q^{2}$ [35] leading to a reconstructed transfer function $\widehat{H}(\omega)$ which does not exhibit unruly behavior outside the high-SNR band. The value chosen for $Q^{2}$ was $0.01 \max (|F(\omega)|)$ [36]. Compared with the transfer function $H(\omega)$ setting $Q^{2}=0$, i.e. in the absence of Wiener filtering, the noise outside the high-SNR band is regularized by removing the anomalous features in the low-SNR bands of $H(\omega)$.

In order to eliminate the remaining noise still present after Wiener filtering, wavelet denoising is employed. In the process of wavelet denoising, based on what was found in Ref. [37], symlet (sym4) wavelets were selected with a level of 5 for the wavelet decomposition, as no significant improvement was found for higher levels; the reconstructed $h(t)$ for various SNR are presented in Fig. 3. After deconvolution by Wiener filtering and wavelet denoising, only the first positive and negative echoes can be resolved. Distinguishing the second positive echo, due to the short time interval $30 T_{\mathrm{s}}$ between the first and third echoes, is beyond the capability of this technique. Moreover, the band-limited nature of the Wiener filter broadens the pulses making up $h_{0}(t)$, ultimately obscuring the locations of the various echoes underlying the reconstructed impulse response function $h(t)$. In addition, anomalous features still remain in both the low- and high-frequency regions even after Wiener filtering when SNR is relatively low, and these features introduce pronounced artifacts in $h(t)$. Besides, the desensitizing factor $Q^{2}$ distorts the deconvolved signal somewhat even within the high-SNR band in the low-noise limit.

Autoregressive extrapolation: AR, one category of parametric spectrum estimation methods, was next used to enhance the ability to reconstruct $h(t)$. Unlike the previous approach employing Wiener filtering and wavelet denoising, which discards high-frequency components with low SNR, AR in general aims at recovering the missing frequency components in the low-SNR regions of the spectrum based on the data within high-SNR regions; hence, the transfer function $H(\omega)$ can be estimated well beyond the lowSNR band, and the peaks in $h(t)$ may not be broadened. Compared with nonparametric methods, no windowing of the autocorrelation sequence is applied, less spectral leakage and better frequency resolution with less computational complexity can be achieved. We therefore expect that this approach may enhance the axial resolution.

There are several possibilities concerning how the spectral extrapolation is carried out. In addition to the BM and MCM, described hereabove, the Yule-Walker method and the covariance methods are also commonly employed in spectral estimation [38]. The two latter methods, however, rely on a minimization of the forward prediction error only. Because low SNR regions are found at both ends of the $\mathrm{THz}$ spectrum, it is important to minimize both the forward (towards higher frequencies) and the backward (toward lower frequencies) prediction errors, and not favor one type of error only. We have verified indeed that Yule-Walker and the covariance methods indeed lead systematically to worse results that the Burg and MCM methods. In the following, we will focus on the latter two techniques only.

In order to be able to apply AR/BM, one must be able to identify low- and high-SNR regions. These can be determined by examining the result of the direct division of $R(\omega)$ by $F(\omega)$, in which the low- and high-SNR bands stand out as they contain wild fluctuations corresponding to high- and low-frequency noise, and can thus be separated by inspection. Still, noise remains within the high-SNR band. Wavelet denoising is thus applied first to selectively reduce noise prior to AR. In the wavelet denoising process, symlet (sym4) wavelets are selected with a level of 9 for the wavelet decomposition. Note that wavelet denoising is a selective (and complex) filter that is intended to retain insofar as possible only features resembling $f_{0}(t)$. Thus, apart from removing noiselike features from the transfer function, it retains relevant information throughout the high-SNR region.

Autoregressive extrapolation with the Burg method: Following the wavelet denoising just described, AR based on the highSNR regions is employed. The forward coefficients $a_{k}$ are estimated from fitting the data within the band [0.2 THz, $\left.1.5 \mathrm{THz}\right]$, and the backward coefficients $b_{k}$ are separately estimated (in BM) from fitting the data within $[0.14 \mathrm{THz}, 1.41 \mathrm{THz}$. The order $p$ is determined to minimize AIC. For example, for $\mathrm{SNR}=10 \mathrm{~dB}$, the order is $p=65$. After fitting the AR model as a prediction filter to extrapolate the missing data below $0.14 \mathrm{THz}$ and above $1.5 \mathrm{THz}$, the entire spectrum of $H(\omega)$ can be estimated. The elimination of wild fluctuations in the transfer function outside the high-SNR band and the recovery of data within the low-SNR bands results. Thanks to AIC, over- and under-fitting can be avoided.

Following this procedure, $h(t)$ for a range of SNRs is shown in Fig. 4(a)-(c). The values for the order of the AR process are $p$ $=70,82$, and 100 when $\mathrm{SNR}=20 \mathrm{~dB}, 10 \mathrm{~dB}$, and $5 \mathrm{~dB}$, respectively, unless otherwise noted. Three peaks, corresponding to the three interfaces of the synthesized double-layered structure $h_{0}(t)$, can be found even under relatively high SNR. Shifts of peak positions in $h(t)$ compared with $h_{0}(t)$, are found, however, and are a typical shortcoming of the Burg method [39][40]. Further, Fig. 4(d) illustrates that spurious peaks occur in the AR/BM reconstruction with $p=72$, which is another know shortcoming of BM. Based on Ref.[34], temporal peak splitting is most likely to occur with AR/BM when the SNR and the model order are both high. Spurious peaks could be misinterpreted as additional layers that are not actually present.

Autoregressive extrapolation by the modified covariance method: To overcome these shortcomings, AR/MCM is applied. Owing to the absence of Levinson-Durbin recursion to solve for the model coefficients, higher computational cost is demanded by AR/MCM.

In addition, because there is no guarantee that the system poles lie inside the unit circle, it is crucial to stabilize the AR model. The approach we use is to reflect the instable poles inside the unit circle by applying the following procedure

$$
D_{i}^{\prime}= \begin{cases}D_{i} & \text { for }\left|D_{i}\right| \leq 1 \\ \frac{1}{\left|D_{i}\right|^{2}} D_{i} & \text { for }\left|D_{i}\right|>1\end{cases}
$$


where $D_{i}$ are the poles of the system and $D_{i}$ the updated poles. More details can be found in Ref. [41]. By comparison, AR/BM is computationally more efficient and produces a stable model; a major drawback is, however, that the value of $p$ is difficult to determine and for instance often does not correspond to the minimization of AIC. Consequently, unless we can optimize $p$ with samples of known thickness, it is difficult to assess the accuracy of the approach. On the other hand, AR/MCM is computationally more intensive, but intrinsically may suffer from instabilities. The latter can often be tamed, resulting in AR/MCM's main advantage: since the forward- and backward-prediction errors are minimized simultaneously, with no restriction introduced by an additional constraint, the value of $p$ that minimizes AIC results in much more reliable reconstruction of $h(t)$. Of course, in both cases, it is the presence of noise that introduces difficulties. As discussed below, for high-SNR, AR/BM is much more likely to be successful.

The corresponding results based on AR/MCM for various SNR are shown in Fig. 5. The values of the order of the AR process are $p=60,55$, and 65 when $\mathrm{SNR}=20 \mathrm{~dB}, 10 \mathrm{~dB}$, and $5 \mathrm{~dB}$, respectively. Compared with the AR/BM reconstructions (see Fig. 4), even though a significant improvement results from AR/MCM and AR/BM with regard to the ability to reconstruct optically thin layer thicknesses, fewer erroneous shifts in the peak positions in $h(t)$ result from applying AR/MCM, suggesting an advantage of the latter approach in that it is not as sensitive to residual noise even after wavelet denoising. In addition, AR/MCM does not suffer from spurious peaks in $h(t)$ under various SNR conditions; AR/MCM thus reliably provides a more accurate reconstruction of the stratigraphy. As for the AM/BM results, those spurious peaks in the reconstructed $h(t)$ may be erroneously interpreted as additional layers that are not actually present.

Because minimizing AIC with respect to $p$ is still likely to result in artifacts in $h(t)$ and finding the optimal value of $p$ may require optimization with a sample of known thickness for AR/BM, we conclude that AR/MCM provides a significantly more reliable approach to finding the optimal order $p$ and the reconstruction $h(t)$ is much less likely to suffer from the artifacts encountered in the AR/BM reconstruction in the presence of noise.

Experimental verification: Having compared the performance of AR/MCM using synthetic data, we next test the approach using experimental THz TOF tomography data from a single layer of mill scale on a steel coupon.

To return to the experiments and reconstruction carried out here, the nominal thickness of the scale layer investigated is characterized independently by a destructive cross-sectional measurement and was found to be $18.5 \mu \mathrm{m}$. The composition of the scale layer is also identified by $\mathrm{x}$-ray diffraction. Based on the Rietveld refinement principle, the concentrations of the various iron-oxide phases present in the mill scale are shown in Fig. 6 showing wüstite to be dominant, with mass percentage $58 \%$ and magnetite (a conductor) the second most dominant phase. Based on this information, we estimate that the refractive index of the scale film is approximately that of wüstite, 4.7 [42]. (We do expect the other iron-oxide phases present to contribute mainly to attenuation, which given the small thickness of the film is not of great importance to the measurement.)

A typical experimental reflected $\mathrm{THz}$ signal at a representative position on the coupon is shown in Fig. 7(a). Due to the fact that the mill scale is thinner than the naïve axial resolution, the echoes reflected from the air/mill-scale and the mill-scale/steel interfaces temporally overlap. Wiener filtering combined with wavelet denoising, AR/BM, and AR/MCM are employed to characterize the thin film, and the corresponding results are also shown in Fig. 7(b)-(d), respectively.

The reconstruction by Wiener filter and wavelet denoising only has one peak, and it therefore is entirely inadequate to recover the two echoes from the air/mill scale and mill scale/steel interfaces, and thus to reconstruct the stratigraphy of the film.

Both AR/BM and AR/MCM provide reconstructions showing two peaks, and the coating thickness estimated based on the optical delay between the two positive peaks is $17.7 \mu \mathrm{m}$ and $18.4 \mu \mathrm{m}$ for AR/BM and AR/MCM, respectively. The former value exhibits a $\sim 10 \%$ error (too large) while the latter shows no significant error. Not shown here, measurements taken at several positions on the sample exhibit the same trends.

In addition, owing to the high sensitivity of AR to noise as well as the AR parameters, we see that peak splitting occurs in $h(t)$ reconstructed by AR/BM if we choose an excessively high order $p$, as shown in Fig. 7(f). Of course, in the present case we can choose a more reasonable value of $p$ to avoid peak splitting, but then, the errors discussed above are still present. As for AR/MCM, due to the approach discussed above to guarantee the stability of the AR model and the reliability of choosing $p$ to minimize AIC, line splitting is not observed and the film thickness is quite accurately reconstructed. In conclusion, a more accurate reconstruction based on AR/MCM compared with AR/BM is achieved.

\section{Conclusion}

In this paper, $\mathrm{AR} / \mathrm{MCM}$ is explored to enhance the ability to reconstruct the stratigraphy of samples with optically thin layers, and has been used to analyze pulsed $\mathrm{THz}$ imaging data. We begin by comparing with Wiener filtering combined with wavelet denoising. This method attempts at reducing noise but cannot effectively reconstruct information outside the high-SNR band. The parametric models $\mathrm{AR} / \mathrm{BM}$ and $\mathrm{AR} / \mathrm{MCM}$ aim at precisely this. While $\mathrm{AR} / \mathrm{BM}$ attempts to minimize the prediction errors it must simultaneously satisfying the Levison-Durbin constraint, making it difficult to choose the optimal order $p$ of the AR model. Moreover, AR/BM tends to broaden features in the reconstructed impulse response if the order is reasonable or too low, while may lead to the appearance of erroneous peaks (peak splitting) if the order is too high. AR/MCM, on the other hand, though suffering from possible instability, can in practice be stabilized, and avoids the problems mentioned above with AR/BM. 
We verify the utility of AR/MCM for optically thin layers in the presence of significant noise highlighting its advantages compared with AR/BM using both synthetic and experimental data, and argue that AR/MCM can be more reliable under practical conditions where the structure of the sample unknown and significant noise is present.

Acknowledgements This work was supported in part by of by Conseil Régional Grand Est, ArcelorMittal Maizières Research, and CPER SusChemProc.

\section{References}

[1] J. Dong, A. Locquet, and D.S. Citrin, IEEE J. Sel. Top. Quantum Electron. 23, 1-7 (2017).

[2] W. Tu, S. Zhong, A. Incecik, and X. Fu, Ocean Eng.155, 382-91 (2018).

[3] T. Yasui, T. Yasuda, K. Sawanaka, and T. Araki, Appl. Opt. 44, 6849-56 (2005).

[4] J. Dong, J. B Jackson, M. Melis, D. Giovanacci, G.C. Walker, A. Locquet, J.W. Bowen, and D.S. Citrin, Opt. Express 24 , 26972-85 (2016).

[5] J. B. Jackson, J. Bowen, G. Walker, J. Labaune, G. Mourou, M. Menu, and K. Fukunaga, IEEE. Trans. Terahertz Sci. Technol. 1, 220-31 (2016).

[6] C.L. Koch-Dandolo, T. Filtenborg, K. Fukunaga, J. Skou-Hansen, and P.U. Jepsen, Appl. Opt. 54, 5123-29 (2015).

[7] A.Y. Pawar, D.D. Sonawane, K.B. Erande, and D.V. Derle, Drug Invent. Today 5,157-63 (2013).

[8] M. Naftaly and R.E. Miles, Proc. IEEE. 95, 1658-1665 (2007).

[9] R. Piesiewicz, T. Kleine-Ostmann, N. Krumbholz, D. Mittleman, M. Koch, and T. Kurner, Electron. Lett. 41, 1002-4 (2005).

[10] A. Taschin, P. Bartolini, J. Tasseva, J. Striova, R. Fontana, C. Riminesi, and R. Torre, arXiv preprint arXiv: 1703.01770 (2017).

[11] A.A. Gowen, C.O'Sullivan, and C.P. O’Donnell, Trends Food Sci. Technol. 25, 40-6 (2012).

[12] F. Rutz, M. Koch, S. Khare, M. Moneke, H. Richter, and U. Ewert, J. Infrared Millim. Terahertz. Waves 25, 547-56 (2006).

[13] A.I. Hernandez-Serrano, S.C. Corzo-Garcia, E. Garcia-Sanchez, M. Alfaro, and E. Castro-Camus, Appl. Opt. 53, 7872-6 (2014).

[14] R.M. Woodward, B.E. Cole, V.P. Wallace, R.J. Pye, D.D. Arnone, E.H. Linfield, and M. Pepper, Phys. Med. Biol. 47, 3853 (2002).

[15] R.K.H. Galvao, S. Hadjiloucas, A. Zafiropoulos, G.C. Walker, J.W. Bowen, and R. Dudley, Opt. Lett. 32, 3008-10 (2007).

[16] Y. Chen, S. Huang, and E. Pickwell-MacPherson, Opt. Express 18, 1177-90 (2010).

[17] D.M. Mittleman, R.H. Jacobsen, and M.C. Nuss, IEEE J. Sel. Top. Quantum Electron. 2, 679-92 (1996).

[18] D.M. Mittleman, R.H. Jacobsen, R. Neelamani, R.G. Baraniuk, M.C. Nuss, Appl. Phys. B. 67, 379-90 (1998).

[19]B. Ferguson and D. Abbott, Microelectron. J. 32, 943-53 (2001).

[20] B. Ferguson and D. Abbott, Fluct. Noise Lett. 1, L65-L69 (2001).

[21] J. Dong, P. Pomarede, L. Chehami, A. Locquet, F. Meraghni, N.F. Declercq, and D.S. Citrin, NDT\&E Int. 99, 72-9 (2018).

[22] J. Dong, X. Wu, A. Locquet, and D.S. Citrin, IEEE Trans. Terahertz Sci. Technol. 7, 260-7 (2017).

[23]EM. Stübling, A. Rehn, T. Siebrecht, Y. Bauckhage, L. Öhrström, P. Eppenberger, JC. Balzer, F. Rühli, M. Koch. Scientific reports 9, 1-8 (2019).

[24] J. Dong, A. Locquet, M. Melis, and D.S. Citrin, Sci. Rep. 7, 1-13 (2017).

[25] H. Karsli, J. Appl. Geophys. 59, 324-36 (2006).

[26] T. Miyashita and T. Itaya, High resolution acoustic impulse response in air with spectral extrapolation by linear prediction, 1998 IEEE Untrasonics Symposium. Proceedings 1, 873-6 (1998).

[27] C.A. Zala, I. Barrodale, and K.I. MaRae, In Signal processing and pattern recognition in nondestructive evaluation of materials, ed. C.H. Chen (Springer, Berlin, Heidelberg, 1988), 44, 101-8.

[28] J. Dong, A. Locquet, and D.S. Citrin, Opt. Lett. 42, 1828-31 (2017).

[29] S. Kay and L. Marple, Sources and remedies for spectral line splitting in autoregressive spectrum analysis, ICASSP'79 IEEE International Conference on Acoustics, Speech, and Signal Processing, 4, 151-4 (1979).

[30] B.M. Bell, IEEE Trans. Signal Process 39, 185-189 (1991).

[31] M. Ortigueira, and J. Tribolet, A framework for the evaluation of spectral analysis techniques, ICASSP'84. IEEE International Conference on Acoustics, Speech, and Signal Processing, 9, 247-50 (1984).

[32] M. Zhai, A. Locauqet, and D.S. Citrin, Terahertz nondestructive thickness characterization of optically thin scale on steel, Surf. Coat. Technol. (submitted)

[33] H. Akaike, IEEE Trans. Automat Contr. 19, 716 (1974).

[34] S.M. Alessio, Digital signal processing and spectral analysis for scientists: Concepts and application. (Springer, Turin, 2015).

[35] R. Shibata, Biometrika, 63, 117-26 (1976).

[36] M. Kazubek, IEEE Signal Proc. Lett. 10, 324-26 (2003).

[37] J. Pei, P. Ye, and W. Xie, Optimal wavelet analysis for THz-TDS pulse signals, Proc. SPIE 7277, Photonics and Optoelectronics Meetings (POEM) 2008: Terahertz Science and Technology, 727708 (2009).

[38] E.C. Ifeachor, and B.W. Jervis, Digital signal processing: a practical approach. (Pearson Education, 2002).

[39] S. Beheshti, IEEE Proceeding, ICASSP, 3, 520-3 (2006). 
[40]P. Sheel, R. Mehra, P. Singh. International Journal of Engineering Trends and Technology, 29, 19-22 (2015).

[41] I. Gupta, IEEE Trans. Antennnas. Propag. 42, 1540-5 (1994).

[42] Th. Henning, B. Begemann, H. Mutschke, and J. Dorschner, Astron. Astrophys. Suppl. Ser. 112, 143-9 (1995). 


\section{Figures}

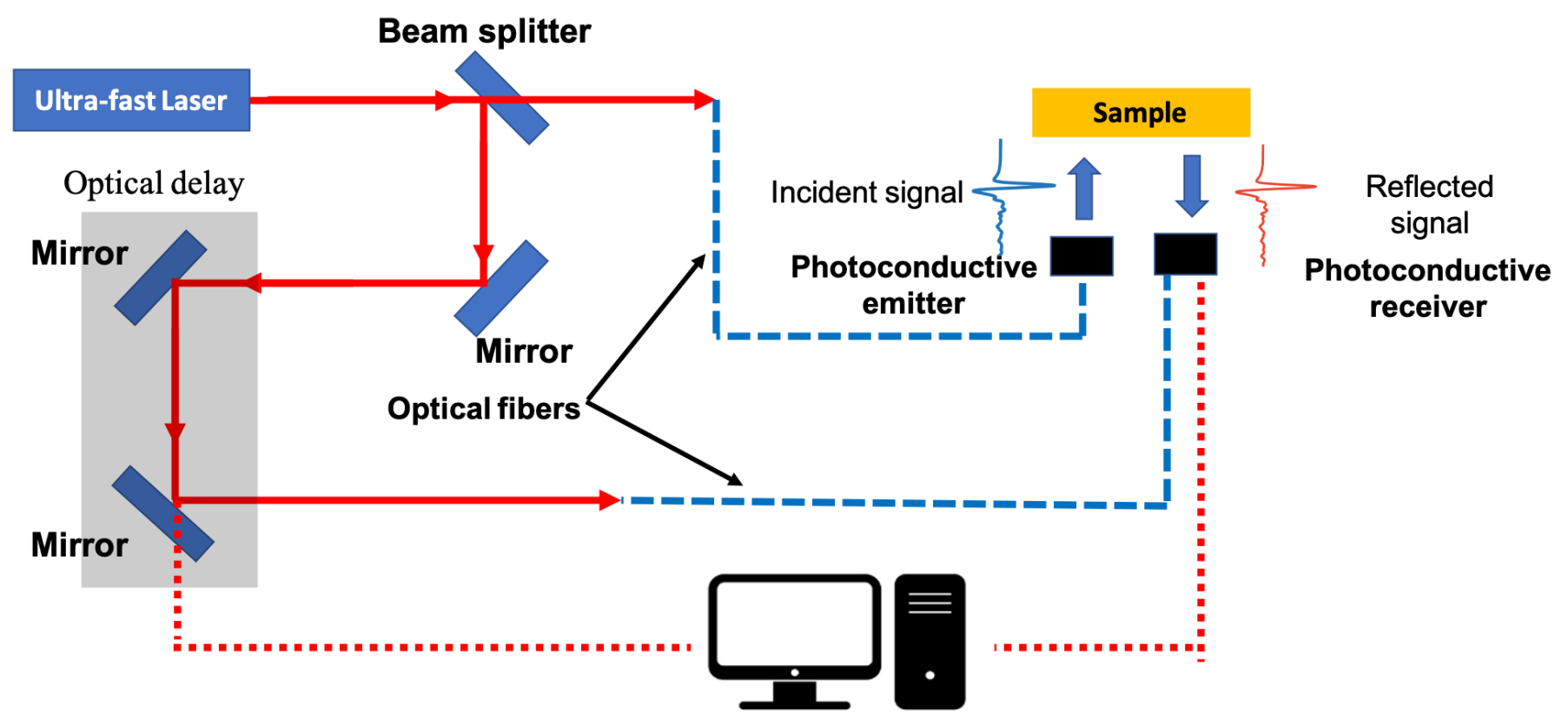

Fig. 1. Schematic diagram of the pulsed THz TDS system. The incident and reflected THz signals impinge at the same point on the sample and are spatially separated in the figure for clarity. 

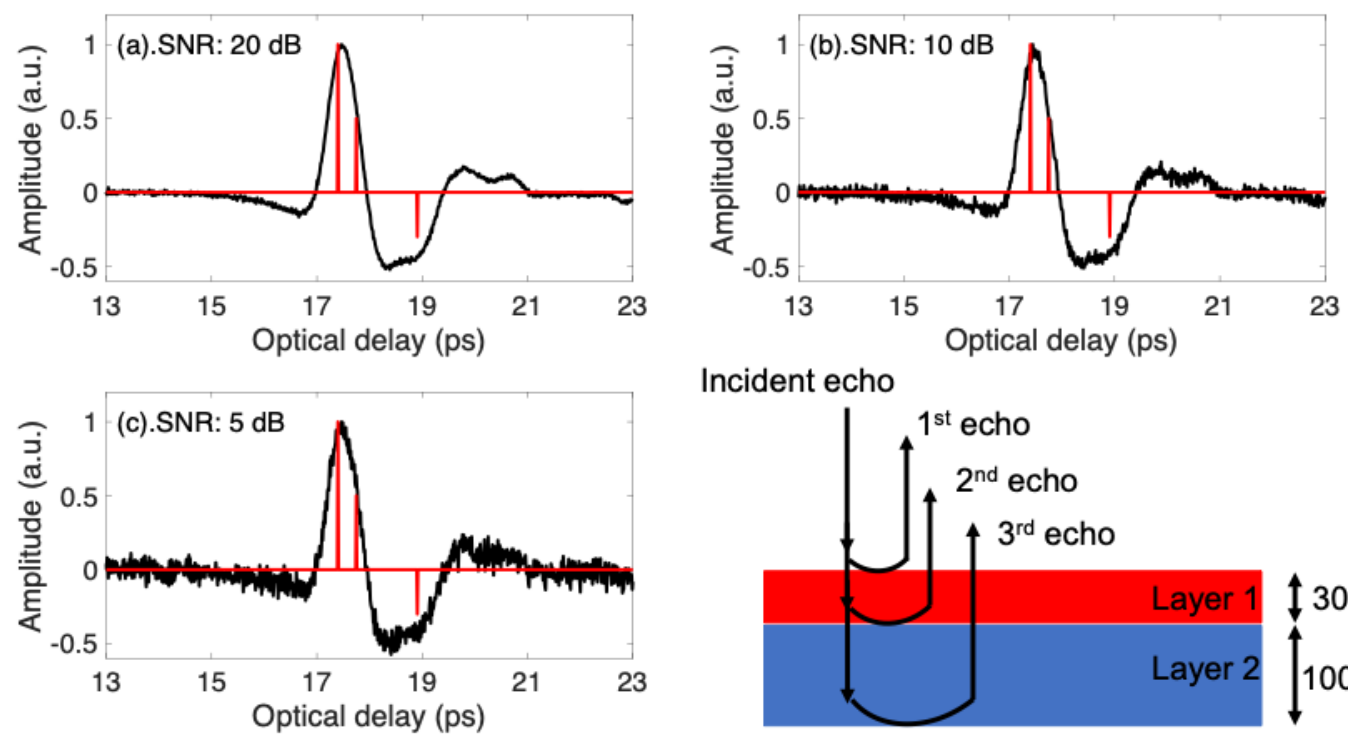

Incident echo

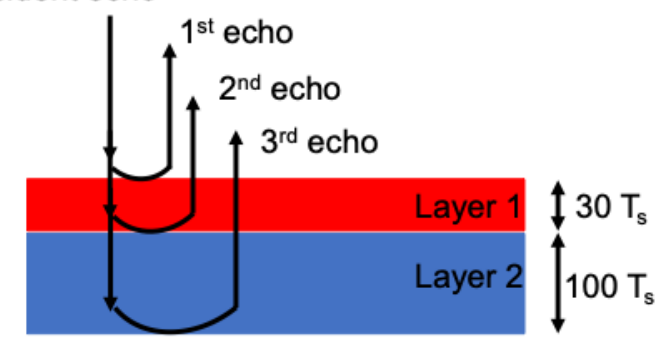

Fig. 2. The synthesized impulse response function (red) $h_{0}(t)$ and computed reflected THz signal (black) $f_{0}(t) \otimes h_{0}(t)+e_{n}(t)$ for various SNRs. The simulated THz signal $r_{0}(t)$ convolves the actual THz reference signal $f_{0}(t)$ with the assumed impulse response function $h_{0}(t)$ with additive Gaussian white noise $e_{n}(t)$. 

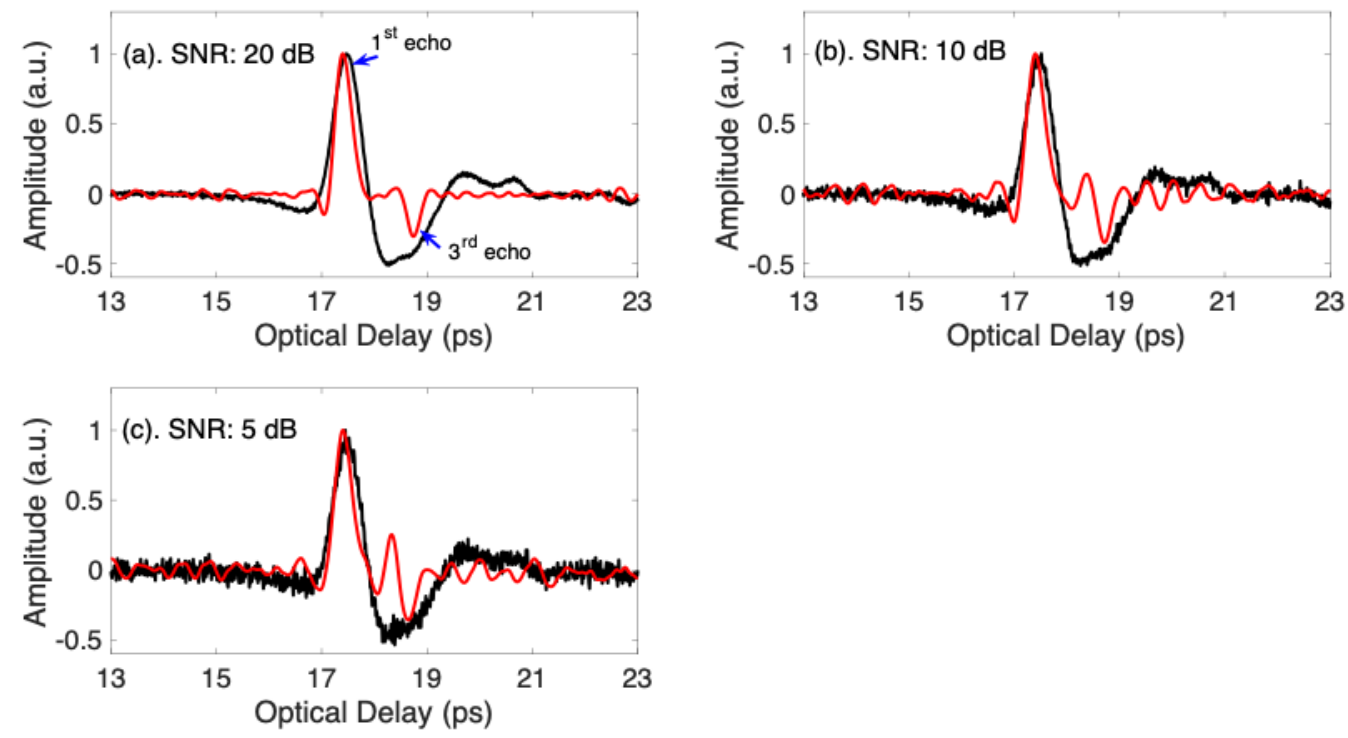

Fig. 3. Black curves show synthesized reflected signals $r_{0}(t)$ at the various noise levels indicated. Reconstruction of $h_{0}(t)$ by Wiener filtering followed by wavelet denoising shown in red. The position of second echo, expected at time delay $17.76 \mathrm{ps}$, is not clearly identified. See Fig. 2 for the corresponding $h_{0}(t)$ and computed reflected THz signals $f_{0}(t) \otimes h_{0}(t)+e_{n}(t)$. 

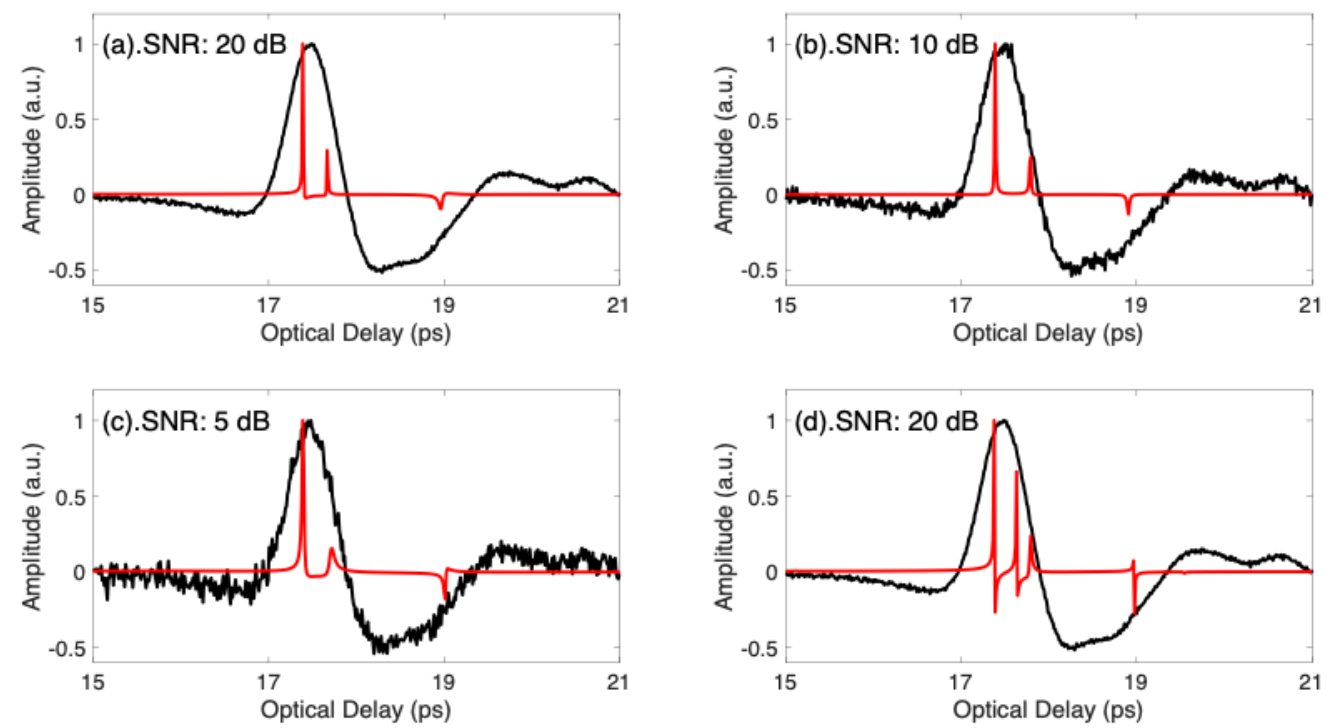

Fig. 4. Comparison of the synthetic reflected $\mathrm{THz}$ signals $f_{0}(t) \otimes h_{0}(t)+e_{n}(t)$ (black) and AR/BM reconstruction $h(t)$ (red) with SNR (a) $20 \mathrm{~dB}$, (b) $10 \mathrm{~dB}$, and (c) $5 \mathrm{~dB}$. (d) Line-splitting [actual peaks in $h_{0}(t)$ appearing as double peaks in $h(t)]$ occurs for $\mathrm{SNR}=20 \mathrm{~dB}$ when an excessively high order is employed, here $p=72$. 

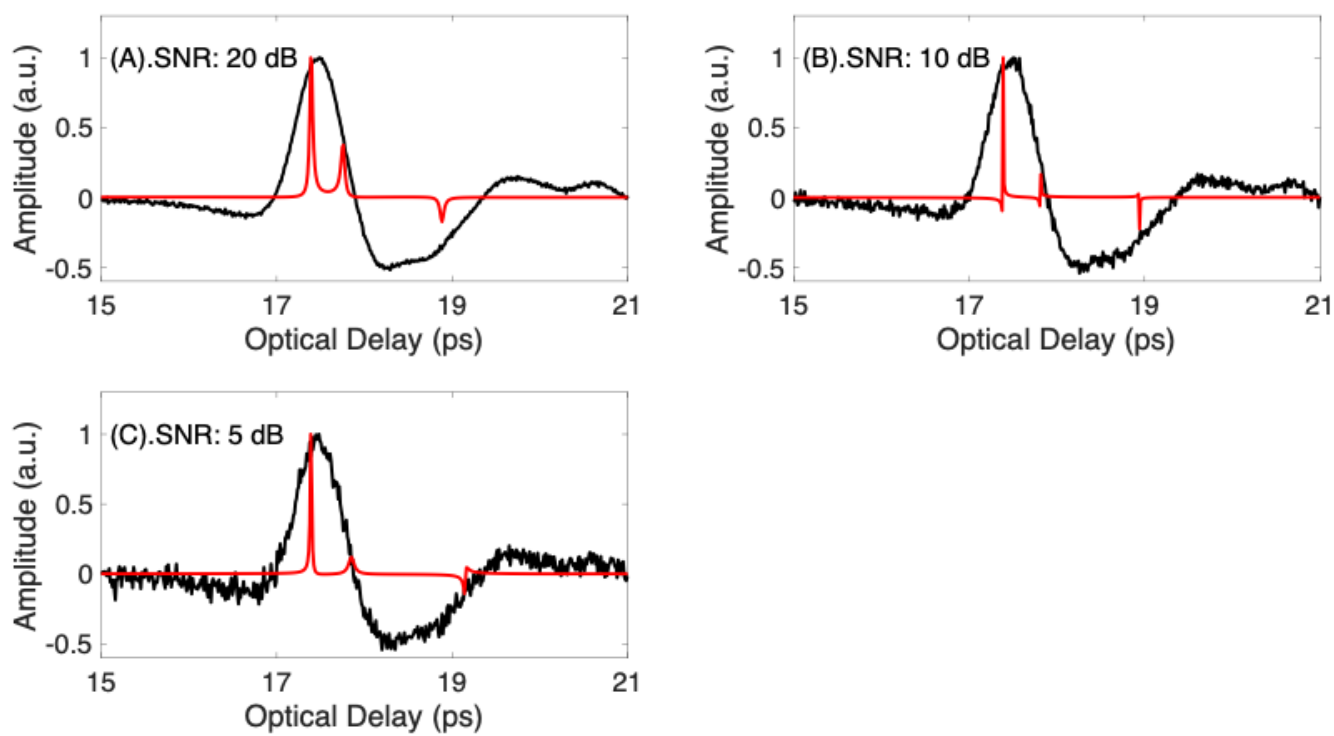

Fig. 5. Comparison of the synthetic reflected $\mathrm{THz}$ signals $f_{0}(t) \otimes h_{0}(t)+e_{n}(t)$ (black) and AR/MCM reconstruction $h(t)$ (red) with SNR (a) $20 \mathrm{~dB}$, (b) $10 \mathrm{~dB}$, and (c) $5 \mathrm{~dB}$. Note that erroneous peaks in $h_{0}(t)$ in Fig. 4(d) are not present here. 


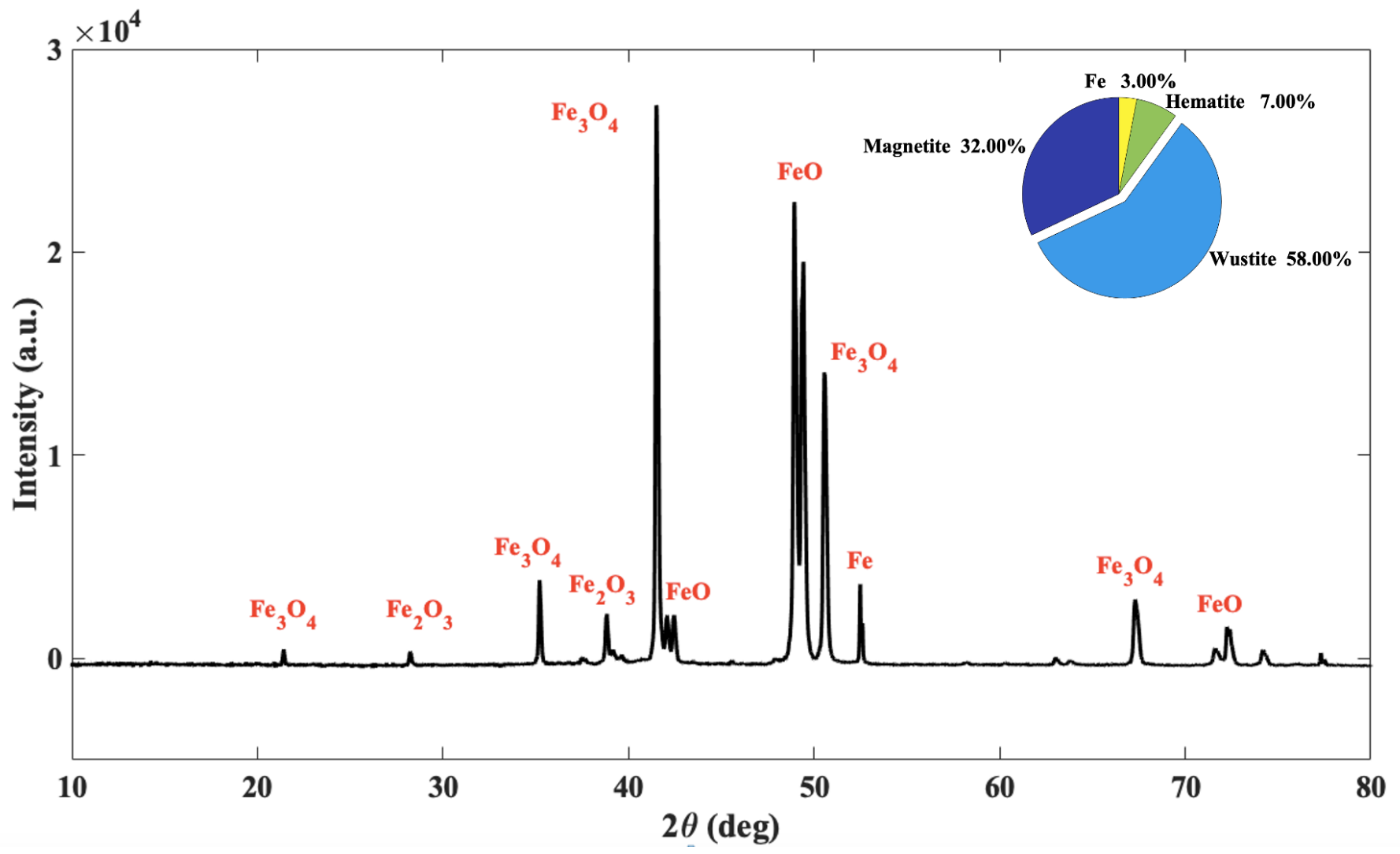

Fig. 6. X-ray diffractogram of $18.5-\mu \mathrm{m}$ thick mill-scale layer on steel substrate. The inset shows the estimated proportion of various iron oxides in the $18.5-\mu \mathrm{m}$ film. 

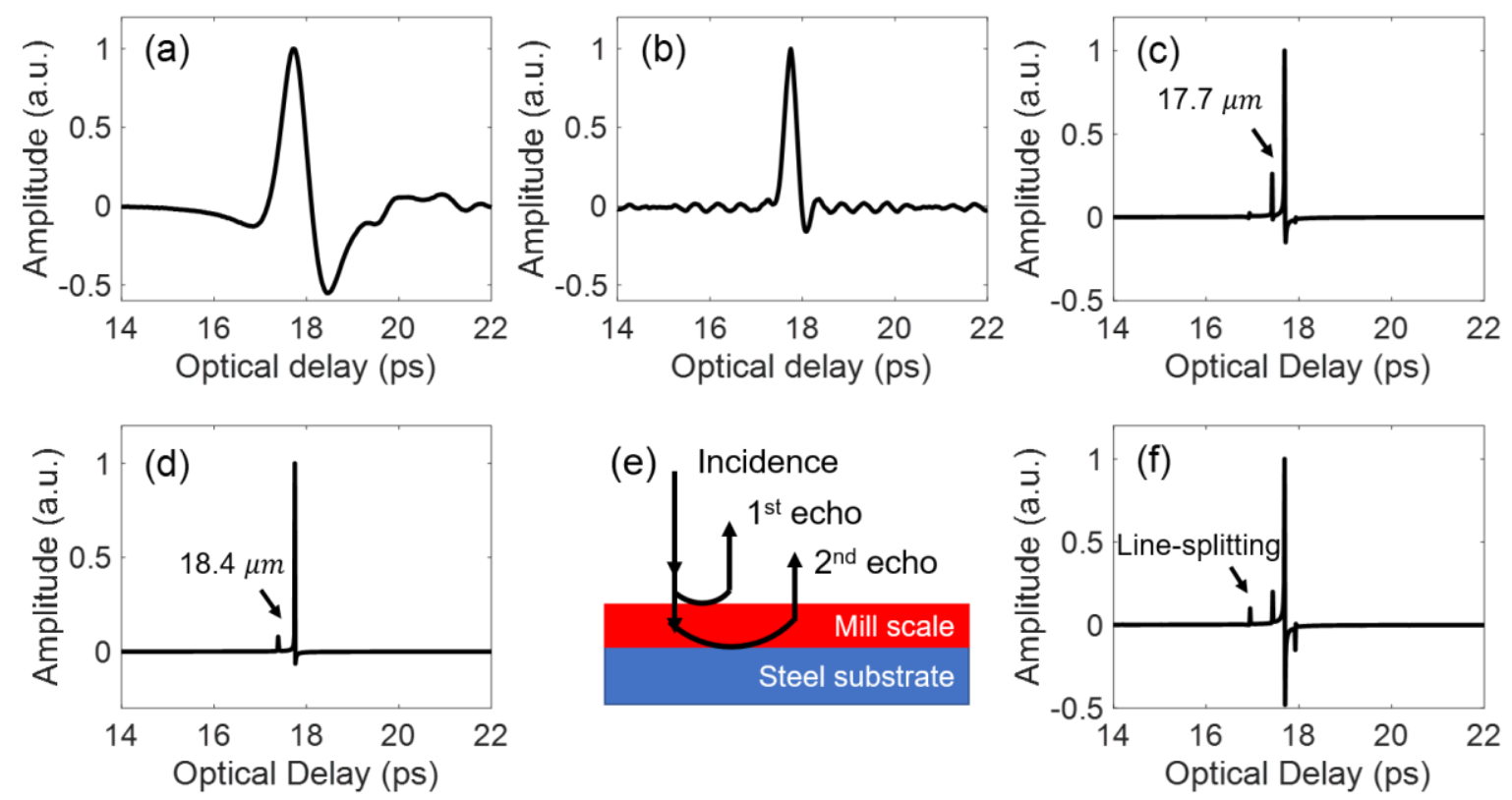

Fig. 7. (a) Raw experimental reflected $\mathrm{THz}$ signal for $18.5-\mu \mathrm{m}$ thick mill scale on steel and the reconstructed impulse-response function $h(t)$ based on (b) Wiener filtering combined with wavelet denoising, (c) AR/BM $(p=75)$, (d) AR/MCM ( $p=60)$, with the corresponding representation of round-trip echoes in (e). (f) Peak splitting occurs in AR/BM when an excessively high order $p$ is chosen $(p=98)$. 